\title{
Turbulent heating and cross-field transport near the magnetopause from THEMIS
}

\author{
C. Chaston, ${ }^{1}$ J. Bonnell, ${ }^{1}$ J. P. McFadden, ${ }^{1}$ C. W. Carlson, ${ }^{1}$ C. Cully, ${ }^{2}$ O. Le Contel, ${ }^{3}$ \\ A. Roux, ${ }^{3}$ H. U. Auster, ${ }^{4}$ K. H. Glassmeier, ${ }^{4}$ V. Angelopoulos, ${ }^{5}$ and C. T. Russell ${ }^{5}$ \\ Received 11 February 2008; revised 28 March 2008; accepted 2 April 2008; published 6 May 2008.
}

[1] We demonstrate that the low frequency broadband magnetic fluctuations observed during THEMIS spacecraft traversals near the Earth's magnetopause may be described as a turbulent spectrum of Doppler shifted kinetic Alfvén waves (KAWs). These waves are most intense along reconnected flux-tubes in the magnetosheath just outside the magnetopause. We identify distinct power-law scalings of wave spectral energy density in wavenumber and show that Landau (LD) and transit time damping (TD) on ions and electrons is largest at the wavenumber where the power-law index changes. The threshold amplitude for stochastic ion scattering/acceleration is also exceeded by these waves. These acceleration processes are manifest in observations of field-aligned and transverse heating of electrons and ions respectively. From integration over the range of observed wavenumbers we show that, if the wave-normal angles are sufficiently large, these waves can provide diffusive transport of magnetosheath plasmas across the magnetopause at up to the Bohm rate. Citation: Chaston, C., et al. (2008), Turbulent heating and cross-field transport near the magnetopause from THEMIS, Geophys. Res. Lett., 35, L17S08, doi:10.1029/ 2008 GL033601.

\section{Observations}

[2] The presence of broadband magnetic noise at the magnetopause has been reported since the first traversals of this boundary by the ISEE spacecraft [Rezeau et al., 1989]. It has been suggested that these oscillations are kinetic Alfvén waves (KAWs) [Johnson et al., 2001; Stasiewicz et al., 2001]. Particle observations in these regions reveal anisotropic ion [Nishino et al., 2007] and field-aligned electron $\left(\mathrm{e}^{-}\right)$distributions [Chaston et al., 2007]. Just inside the magnetopause, spacecraft observe a boundary layer of mixed magnetosheath/magnetospheric like plasmas [Song et al., 2003]. The role that KAWs may play in particle acceleration/heating and transport across the magnetopause to account for these observations has been considered [Lee et al., 1994; Johnson and Cheng, 1997,

\footnotetext{
${ }^{1}$ Space Science Laboratory, University of California, Berkeley, California, USA

${ }^{2}$ Laboratory for Atmospheric and Space Physics, University of Colorado, Boulder, Colorado, USA.

${ }^{3}$ Centre d'Etude des Environnements Terrestre et Planétaires, Velizy, France.

${ }^{4}$ Institut für Geophysik und Extraterrestrische Physik, Technische Universität Braunschweig, Braunschweig, Germany.

${ }^{5}$ Institute for Geophysical and Planetary Physics, University of California, Los Angeles, California, USA.
}

Copyright 2008 by the American Geophysical Union. 0094-8276/08/2008GL033601
2001; Chaston et al., 2007]. In this report we exploit the multi-point measurements provided by the THEMIS spacecraft to characterize the low frequency electromagnetic oscillations in the near magnetosheath/magnetopause/ boundary layer and discuss how these oscillations may drive particle acceleration and cross-field transport in these regions.

[3] Figure 1 presents observations recorded during a THEMIS [Angelopoulos, 2008] traversal of the magnetopause. Measurements from the ion plasma instrument [McFadden et al., 2008] shown in Figure 1a reveal the shocked solar wind plasmas of the magnetosheath transitioning to the more energetic plasmas of the magnetosphere after 0300 UT via a region of mixed magnetosheath/magnetospheric plasmas comprising the low latitude boundary layer. Figure $1 \mathrm{~b}$ reveals a significant density gradient across this transition which provides increasing Alfvén speed $\left(\mathrm{V}_{\mathrm{A}}\right)$ with decreasing flow speed (Figure 1c) into the boundary layer. Measurements from the fluxgate magnetometer experiment [Auster et al., 2008] shown in Figure 1d indicate that at those times in the magnetosheath when $B_{\text {ZGSE }}$ is negative we find enhanced ion energies in Figure 1a. While not especially clear in this case, during these times in the magnetosheath we generally observe enhanced magnetic fluctuations (see black trace in Figure 1d). Entry into the magnetosphere is coincident with enhanced electric field fluctuations, as shown in Figure 1e, which maximize in the boundary layer before decreasing with penetration deeper into the magnetosphere. With the enhancements in the magnetic field and electric field fluctuations in the magnetosheath and boundary layer respectively we observe fieldaligned and counter-streaming $\mathrm{e}^{-}$bursts as shown in Figures $1 \mathrm{f}$ and $1 \mathrm{~g}$.

[4] Figure 2 presents a 'zoom-in' on enhanced wave activity collected during the 'particle burst' interval highlighted in Figure 1. Figures $2 \mathrm{a}$ and $2 \mathrm{~b}$ show the field quantities in a field-aligned coordinate system where $\mathrm{Y}$ is the component in the spacecraft spin plane perpendicular to $B_{o}$ and $Z$ is field-aligned. These data reveal a steepened magnetic waveform and contain a large compressional magnetic field component $\left(\mathrm{B}_{\mathrm{z}}\right)$. This steepened compressive waveform delineates the commencement of enhanced magnetic and bursty transverse electric field oscillations shown in Figure 2b. These enhanced fluctuation amplitudes are coincident with increases in $\mathrm{T}_{\mathrm{e} \|}$ and $\mathrm{T}_{\mathrm{i} \perp}$ shown in Figure 2c suggestive of wave heating. The contour plots presented in Figures $2 \mathrm{~d}-2 \mathrm{~g}$ show the ion and $\mathrm{e}^{-}$distributions before and during the wave burst. These reveal enhanced $\mathrm{e}^{-}\left(\mathrm{T}_{\perp \mathrm{e}}<\right.$ $\left.\mathrm{T}_{\| \mathrm{e}}\right)$ and ion anisotropy $\left(\mathrm{T}_{\perp \mathrm{i}}>\mathrm{T}_{\| \mathrm{i}}\right)$ during the wave burst and the appearance of enhanced phase space densities at large energies. 


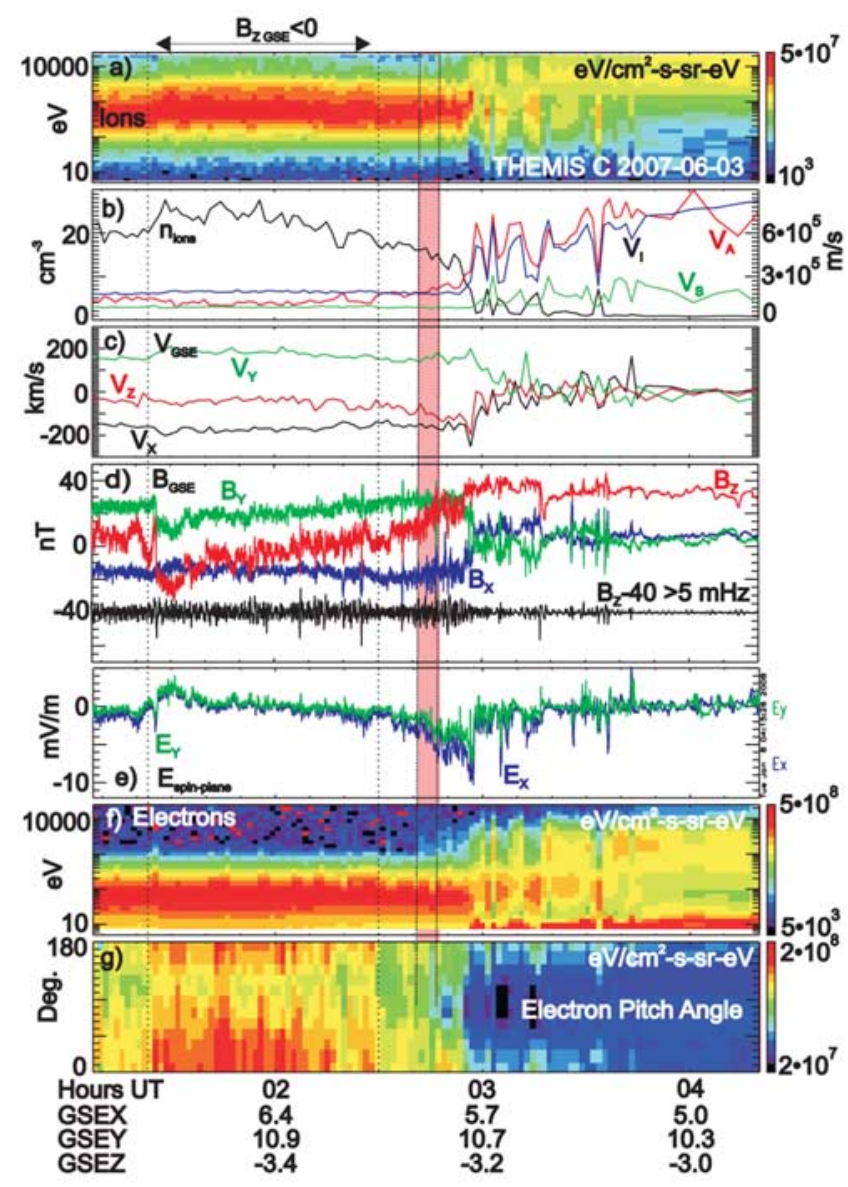

Figure 1. (a) Ion energy spectrogram. (b) Ion density (black), Alfvén speed (red), ion thermal speed (blue) and ion sound speed (green). (c) Ion flow speed in GSE coordinates. (d) Magnetic field in GSE coordinates. (e) Despun spin plane electric field in near GSE coordinates. (f and g) Spectrogram of $\mathrm{e}^{-}$energy and pitch angle. Pink shaded band shows interval of 'particle burst' data capture.

[5] To further characterize these field fluctuations we perform interferometric analyses. Figure 3 a shows the separation $\left(\mathrm{d}_{\mathrm{cd}}\right)$ between spacecraft $\mathrm{C}$ and $\mathrm{D}$ over a second interval where $d_{c d}$ approached an ion gyro-radii during a transition through the magnetopause/sheath. The phase difference in B between spacecraft C and D derived from wavelet based cross-correlation [Chaston et al., 2005] is shown in Figure $3 \mathrm{~d}$. The increasing phase $\left(\phi_{\mathrm{cd}}\right)$ with increasing spacecraft frame wave frequency $\left(\mathrm{f}_{\mathrm{sf}}\right)$ revealed here is indicative of increasing wavenumber $\mathrm{k}_{\mathrm{cd}}=\phi_{\mathrm{cd}} / \mathrm{d}_{\mathrm{cd}}$ along this baseline with $\mathrm{f}_{\mathrm{sf}}$. Following $1500 \mathrm{UT}$ 'antennae nulls' are apparent as $\phi_{\mathrm{cd}}$ passes through $-\pi,-3 \pi,-5 \pi$. The average $\mathrm{k}_{\mathrm{cd}}$ determined by this technique is shown as a function of $\mathrm{f}_{\mathrm{sf}}$ in Figure 3e. Alternatively, if $\omega / \mathrm{k}$ is much less than the flow speed, $\mathrm{k}$ may be estimated along the flow vector $\mathbf{v}_{\mathrm{p}}$ from the resultant Doppler shift in the spacecraft frame. In this case the angular frequency in the spacecraft frame is $\omega_{\mathrm{sf}}=\omega+\mathbf{k} \cdot \mathbf{v}_{\mathrm{p}} \approx \mathbf{k} \cdot \mathbf{v}_{\mathrm{p}}$. The component of $\mathbf{k}$ along $\mathrm{v}_{\mathrm{p}}$ is then given by $\mathrm{k}_{\mathrm{T}}=\omega_{\mathrm{sf}} / \mathrm{v}_{\mathrm{p}}$ (also known as Taylor's approximation). To test the validity of this approximation we project $\mathbf{k}_{\mathrm{T}}$ along the C-D baseline $\left(\mathrm{k}_{\mathrm{T} \text { proj_CD }}\right)$ and compare it to $\mathrm{k}_{\mathrm{cd}}$. The red line in Figure $3 \mathrm{e}$ represents $\mathrm{k}_{\mathrm{T} \_ \text {proj_CD }}$ and shows good agreement with $\mathrm{k}_{\mathrm{cd}}$ over the range $0.005 \mathrm{~Hz}<\mathrm{f}_{\mathrm{sf}}<0.2 \mathrm{~Hz}\left(\mathrm{f}_{\mathrm{sf}}=\omega_{\mathrm{sf}} / 2 \pi\right)$ where reliable phase measurements are obtained. This provides us with confidence that $\mathrm{k}_{\mathrm{T}}$ is a useful proxy for $\mathrm{k}$.

[6] Under the assumption that Taylor's approximation is also valid for other magnetosheath/sphere traversals with similar flow speeds we now compile spectrograms in $B_{X}$ and $E_{Y}$ averaged over 10 traversals from the period June-August 2007 when the spacecraft were in 'particle burst mode'. In this mode the spacecraft returns waveforms with a Nyquist of $64 \mathrm{~Hz}$. We are unable to confirm the validity of Taylor's approximation up to this Nyquist, however, the wave model we present later suggests that such an assumption is reasonable. These averages are compiled irrespective of IMF orientation, however we note that spectral energy densities are generally larger for those intervals where $\mathrm{B}_{\mathrm{ZGSE}}$ is negative. The result for $\mathrm{B}_{\mathrm{X}}$ is shown in Figure $4 \mathrm{a}$ and is composed of measurements performed by the fluxgate (black) and search coil (A. Roux et al., Searchcoil magnetometer for THEMIS, submitted to Space Science Reviews, 2008) (blue) magnetometers on THEMIS C. The frequency range over which each instrument provides reliable spectral estimates is shown by the solid lines. We find that the spectral form obtained in this averaged case is very similar to that obtained where Taylor's approximation was checked via interferometry and note that because $\omega_{\mathrm{sf}}$ and $\mathrm{k}_{\mathrm{T}}$ are

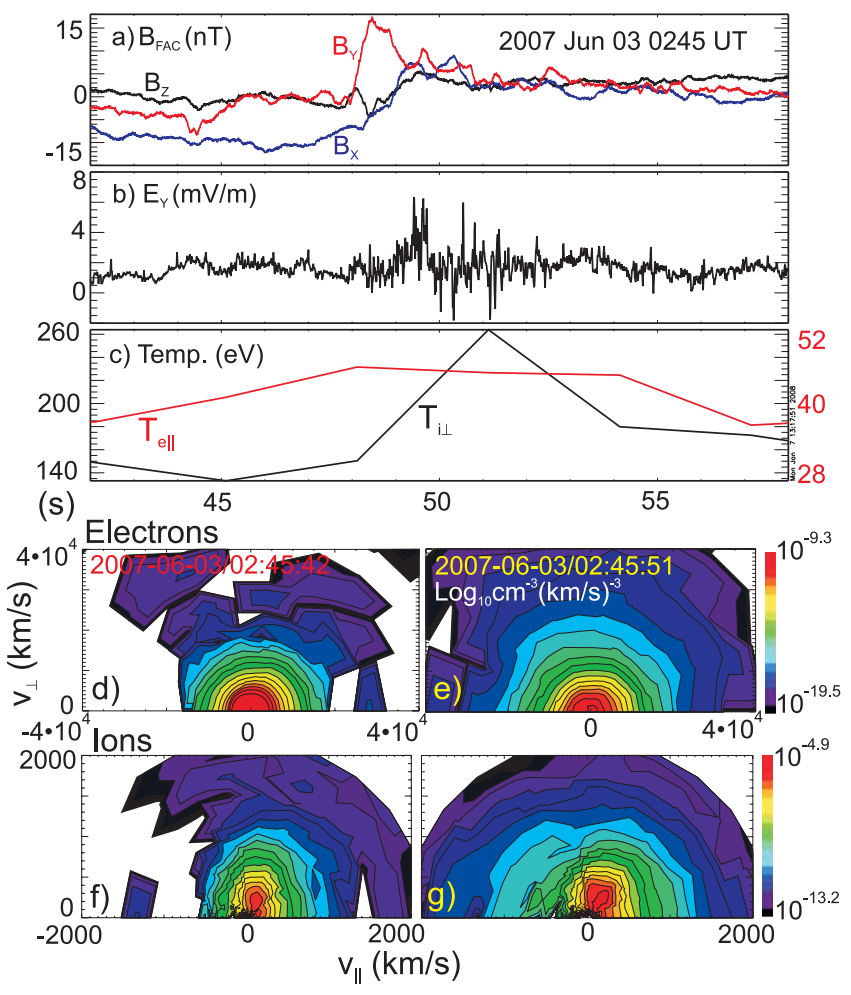

Figure 2. (a) Wave magnetic field in field-aligned coordinate system (FAC) defined in the text. (b) Perpendicular electric field component in FAC. (c) Perpendicular $(\perp$ ) and parallel $(\|)$ ion and $\mathrm{e}^{-}$temperatures respectively. Also shown are $\mathrm{e}^{-}$and ion distribution functions ( $\mathrm{d}$ and $\mathrm{f}$ ) preceding and (e and g) during the wave burst. 


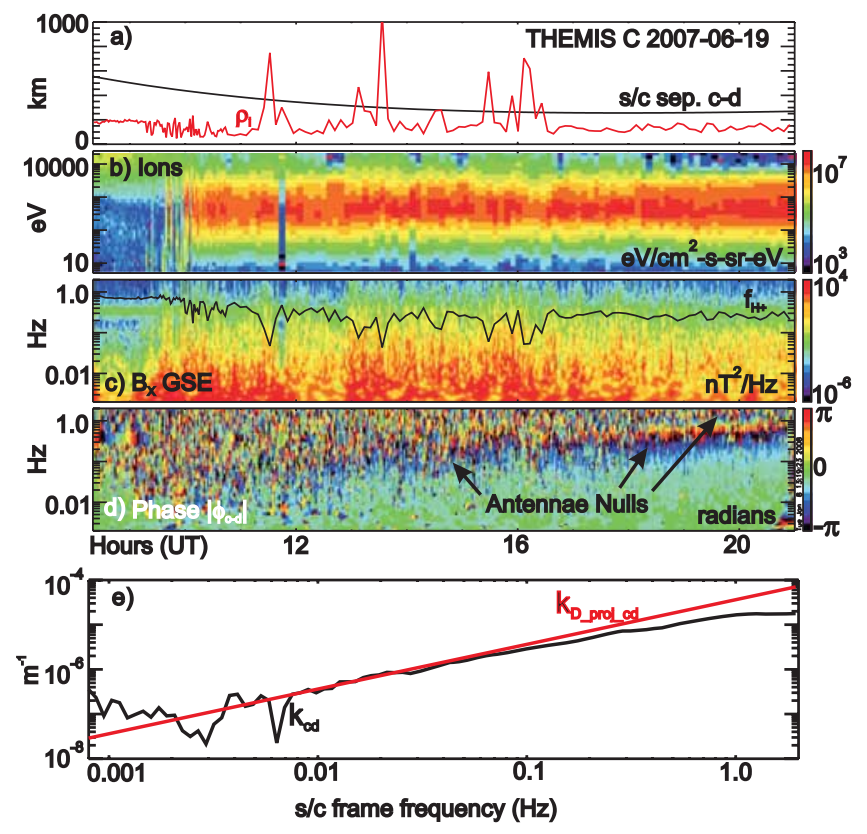

Figure 3. (a) Separation between spacecraft C and D and ion gyro-radii. (b) Ion energy spectrogram. (c) Spectral energy density in $B_{x}$ GSE from fluxgate magnetometer. (d) Modulus phase difference measured between spacecraft $\mathrm{c}$ and $\mathrm{d}$. (e) $\mathrm{k}$ along spacecraft $\mathrm{c}-\mathrm{d}$ baseline and that given by Doppler shift projected along the c-d baseline.

linearly related that the scaling in $\mathrm{nT}^{2} / \mathrm{Hz}$ will be equivalent to that obtained in $\mathrm{nT}^{2} /($ radians $/ \mathrm{m})$. The averaged values of the plasma parameters observed are $\mathrm{B}_{\mathrm{o}}=31 \mathrm{nT}, \mathrm{n}_{\mathrm{i}}=$ $21 \mathrm{~cm}^{-3}, \mathrm{~T}_{\mathrm{e}}=37 \mathrm{eV}$, and $\mathrm{T}_{\mathrm{i}}=235 \mathrm{eV}$. Using these values the spectrogram shown in Figure 4a reveals a range of wave scales extending through an ion gyro-radius $\left(\rho_{\mathrm{i}}\right)$, ion inertial length $\left(\lambda_{\mathrm{i}}\right)$, ion acoustic gyro-radius $\left(\rho_{\mathrm{s}}\right)$ and down to $\mathrm{e}^{-}$ inertial lengths $\left(\lambda_{e}\right)$. The wave spectra over this range contain two distinct ranges which can be approximated by power-laws varying as $\mathrm{k}_{\mathrm{T}}^{-1.5}$ and $\mathrm{k}_{\mathrm{T}}^{-3.0}$ with the break-point between slopes at $\mathrm{k}_{\mathrm{T}} \rho_{\mathrm{s}} \approx 1$. A different scaling is observed in the electric field measurements which decrease more slowly with increasing wavenumber as shown by the red trace in Figure 4a. These results provide an increasingly electrostatic wave with increasing $\mathrm{k}_{\mathrm{T}}$.

[7] To understand the nature of these field fluctuations we implement a wave model appropriate for $\omega \ll \Omega_{\mathrm{p}}$ and $\mathrm{m}_{\mathrm{e}} / \mathrm{m}_{\mathrm{i}}<\beta_{\mathrm{e}}<1$ which includes ion motion along $\mathrm{B}_{\mathrm{o}}$ [Chaston et al., 2005]. In the homogeneous case the wave dispersion is given by the solution of,

$$
k_{z}^{2} v_{A}^{2} k_{y}^{2} \rho_{s}^{2}=\left(\omega^{2}-\frac{k_{z}^{2} v_{A}^{2}}{\chi_{i}}\right) \cdot\left[1-\frac{k_{z}^{2} v_{s}^{2}}{\omega^{2}} I_{o}\left(\alpha_{i}\right) e^{-\alpha_{i}}\right]
$$

where $\chi_{i}=\left[1-I_{o}\left(\alpha_{i}\right) e^{-\alpha_{i}}\right] / \alpha_{i}, \alpha_{\mathrm{i}}=\mathrm{k}_{\mathrm{y}}^{2} \rho_{\mathrm{i}}^{2}, \mathrm{v}_{\mathrm{s}}$ is the acoustic speed, and $\mathrm{I}_{\mathrm{o}}$ is the zero order modified Bessel function. This equation has solutions corresponding to the KAW and ion acoustic wave. Significantly, the wave carries a parallel electric field given by,

$$
\frac{E_{z}}{E_{y}}=-\frac{k_{z} k_{y} \rho_{s}^{2} \chi_{i}}{1-I_{o}\left(\alpha_{i}\right) e^{-\alpha_{i}} \frac{k_{z}^{2} v_{s}^{2}}{\omega^{2}}}
$$

[8] We also note that at large values of $\beta$ the wave carries an appreciable compressional magnetic field component. These waves can be identified from THEMIS by the

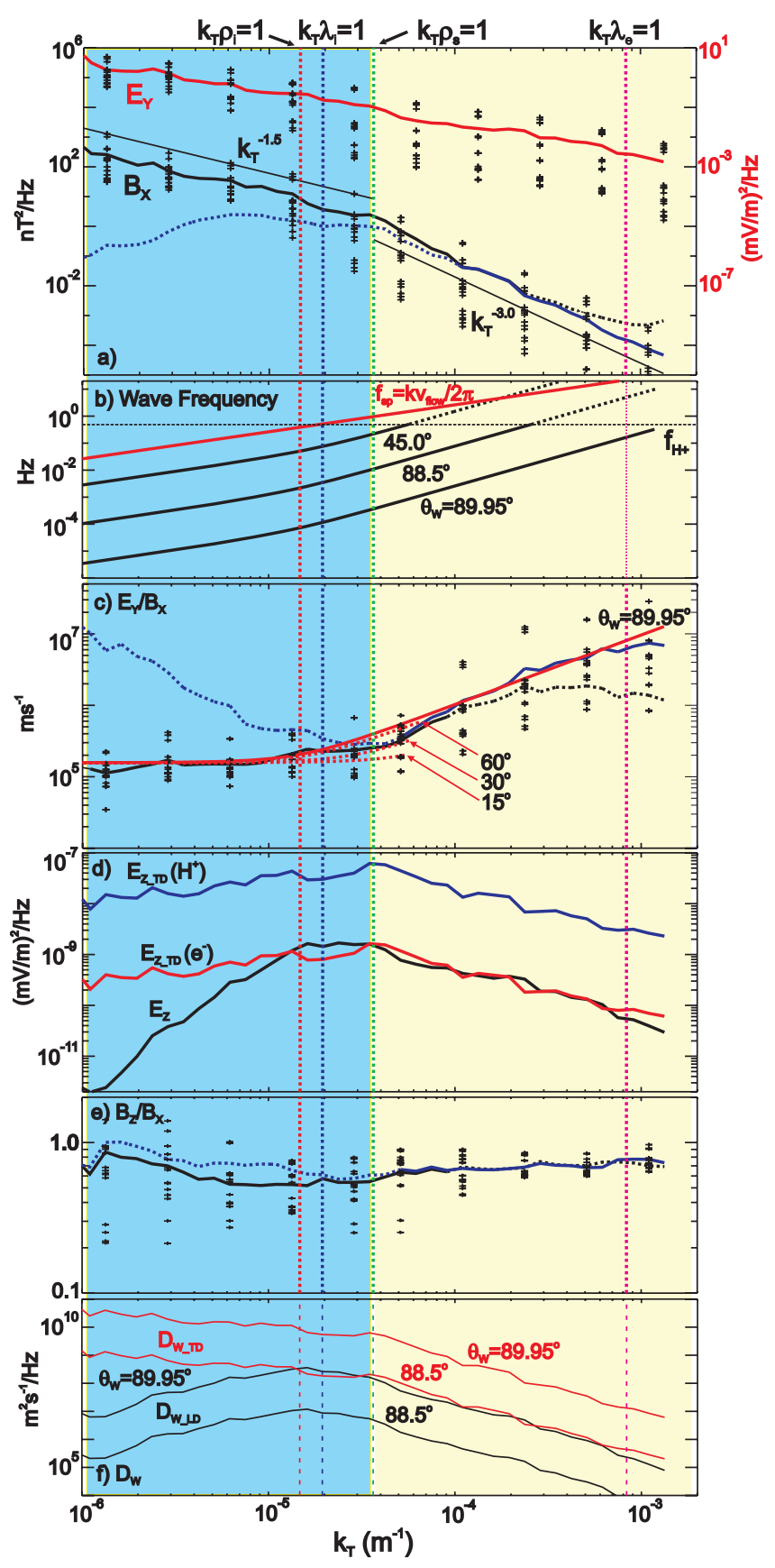

Figure 4. (a) Transverse electric $\left(E_{Y}\right)$ and magnetic $\left(B_{X}\right)$ field spectrograms. Blue and black traces show search coil and fluxgate magnetometer results respectively. The crosses show data points for individual burst intervals. (b) Wave frequency $(\omega)$ from solution of equation (1) for wavenormal angles $\left(\theta_{\mathrm{W}}\right)$ shown. (c) Observed $\mathrm{E}_{\mathrm{X}} / \mathrm{B}_{\mathrm{Y}}$ ratio and model results from equation (3). (d) Parallel electric field $\left(E_{Z}\right)$ from equation (2) and effective field ( $\left.\mathrm{E}_{Z_{\mathrm{T}} \mathrm{TD}}\right)$ due to mirror force on $\mathrm{e}^{-}$and ions. (e) Compressional $\left(\mathrm{B}_{\mathrm{Z}}\right)$ to transverse magnetic field amplitudes. (f) Diffusion coefficient from equation (4). 
comparison of the observed $\mathrm{E}_{\mathrm{y}} / \mathrm{B}_{\mathrm{x}}\left(\mathrm{k}_{\mathrm{T}}\right)$ ratio with the expected ratio given by the expression

$$
\frac{E_{y}}{B_{x}}=-\frac{\omega}{k_{z}}\left[1+\frac{k_{y}^{2} \rho_{s}^{2} \chi_{i}}{1-I_{o}\left(\alpha_{i}\right) e^{-\alpha_{i}} \frac{k_{z}^{2} v_{s}^{2}}{\omega^{2}}}\right]^{-1}
$$

[9] To perform this comparison requires both $\mathrm{k}_{\mathrm{y}}$ and $\mathrm{k}_{\mathrm{z}}$. While it seems likely that $\mathrm{k}_{\mathrm{T}} \sim \mathrm{k}, \mathbf{k}_{\mathrm{T}}$ and $\mathbf{k}$ are not necessarily aligned. Figure $4 \mathrm{~b}$ shows $\omega$ from the solution of 1 for a number of wavenormal angles given by $\theta_{\mathrm{W}}=$ $\arctan \left(\mathrm{k}_{\mathrm{y}} / \mathrm{k}_{\mathrm{z}}\right)$. These show increasing wave frequency with increasing $\mathrm{k}_{\mathrm{T}}$ and decreasing $\theta_{\mathrm{W}}$. The spacecraft frame frequency $\omega_{\text {sf }}$ given by Doppler shift is indicated by the red line and lies above $\omega$ for the entire range in $\mathrm{k}_{\mathrm{T}}$ for $\theta_{\mathrm{W}} \geq$ $80^{\circ}$. Figure $4 \mathrm{c}$ shows that the observed $\mathrm{E}_{\mathrm{y}} / \mathrm{B}_{\mathrm{x}}\left(\mathrm{k}_{\mathrm{T}}\right)$ (black blue) is well matched by the predicted ratio from equation (3) (red). Each red trace here corresponds to a different $\theta_{\mathrm{W}}$ and is plotted for $\mathrm{k}_{\mathrm{T}}$ where $\omega<\Omega_{\mathrm{p}}$. Significantly, the range of $\theta_{\mathrm{W}}$ which provides $\mathrm{E}_{\mathrm{y}} / \mathrm{B}_{\mathrm{x}}$ ratios similar to the observed value contracts toward a value of $90^{\circ}$ with increasing values of $\mathrm{k}_{\mathrm{T}}$. This suggests that these waves become increasingly oblique with increasing $\mathrm{k}$. We also note that the range of $\omega$ that provide $\mathrm{E}_{\mathrm{y}} / \mathrm{B}_{\mathrm{x}}$ values similar to the observed ratio satisfy $\omega \ll \omega_{\text {sf }}=k_{\mathrm{T}} \mathrm{V}_{\mathrm{p}}$ as required for the validity of Taylor's approximation. This provides confidence that this approximation is appropriate over the observed range in $\omega_{\text {sf }}$.

[10] The black trace in Figure $4 d$ shows the spectral energy density of the parallel electric field as given by equation (2) and the measured spectrum in $\mathrm{E}_{\mathrm{y}}$. In calculating this result we have taken $\theta_{\mathrm{W}}=89.95^{\circ}$ and note that since $\mathrm{E}_{\mathrm{z}} /$ $\mathrm{E}_{\mathrm{y}}$ varies approximately linearly with $\mathrm{k}_{\mathrm{z}}$, smaller $\theta_{\mathrm{W}}$ provide larger parallel fields. $\mathrm{E}_{\mathrm{z}}$ increases with $\mathrm{k}_{\mathrm{T}}$ up to a peak at $\mathrm{k}_{\mathrm{T}} \rho_{\mathrm{s}} \approx 1$ where we observe the breakpoint in the $\mathrm{B}_{\mathrm{X}}$ spectrogram of Figure $4 \mathrm{a}$. This $\mathrm{E}_{\mathrm{Z}}$ can lead to dissipation through Landau damping (LD) [Lysak and Lotko, 1996] and may account for the more rapidly falling spectral energy in densities in $\mathrm{B}_{\mathrm{x}}$ for $\mathrm{k}_{\mathrm{T}} \rho_{\mathrm{s}} \geq 1$ Using equation (2) and the observed spectral energy densities in $\mathrm{E}_{\mathrm{y}}$ for $\mathrm{k}_{\mathrm{T}} \rho_{\mathrm{i}} \geq 1$ we find that the field-aligned potential over $1 / 2$ wavelength is $\mathrm{V}_{\mathrm{Z}}=$ $\int_{0}^{\pi} E_{z} / k_{z} d \theta \approx 10 \mathrm{~V}$. Since $\mathrm{eV}_{\mathrm{Z}}$ is a significant fraction of the $\mathrm{e}^{-}$temperature and the ion thermal speed $\mathrm{v}_{\mathrm{i}} \sim \mathrm{v}_{\mathrm{A}}$ it is probable that particle acceleration through LD is occurring for $\mathrm{k}_{\mathrm{T}} \rho_{\mathrm{s}} \geq 1$ and may account for the parallel energization observed.

[11] Alternatively, the large $\mathrm{B}_{\mathrm{z}}$ carried by these waves may provide parallel acceleration through transit time damping (TD). Figure $4 \mathrm{e}$ shows the observed $\mathrm{B}_{\mathrm{z}} / \mathrm{B}_{\perp}$ ratio (black/ blue). From the ion momentum equation and Ampere's law this can be estimated for KAWs as $\mathrm{B}_{\mathrm{Z}} / \mathrm{B}_{\mathrm{X}} \approx \mathrm{i} \beta\left(\mathrm{k}_{\mathrm{y}} / \mathrm{k}_{\mathrm{z}}\right)\left(\omega / \Omega_{\mathrm{i}}\right)$ $\chi_{\mathrm{i}}\left[1-\left(\mathrm{k}_{\mathrm{z}}^{2} \mathrm{v}_{\mathrm{s}}^{2} / \omega^{2}\right) \mathrm{I}_{\mathrm{o}}\left(\alpha_{\mathrm{i}}\right) \mathrm{e}^{-\alpha \mathrm{i}}+\mathrm{k}_{\mathrm{y}}^{2} \rho_{\mathrm{s}}^{2} \chi_{\mathrm{i}}\right]^{-1}$ which for $\mathrm{k}_{\mathrm{y}} \rho_{\mathrm{i}} \geq 1$ and $\theta_{\mathrm{W}}>15^{\circ}$ provides values of $\mathrm{B}_{\mathrm{Z}} / \mathrm{B}_{\mathrm{X}}$ between 0.5 and $\sim 1$. The relative importance of $\mathrm{LD}$ and TD can be assessed by comparing $\mathrm{E}_{\mathrm{Z}}$ as calculated from equation (2) with an effective 'parallel electric field' $\left(\mathrm{E}_{\mathrm{z}} \mathrm{TD}\right)$ associated with the mirror force. This is given by $\mathrm{E}_{\mathrm{Z}_{-} \mathrm{TD}}=-\left[\mathrm{m}_{\mathrm{j}} \mathrm{v}_{\mathrm{j} \perp}^{2} /\left(2 \mathrm{~B}_{\mathrm{o}}\right)\right]$ $\mathrm{ik}_{\mathrm{Z}} \mathrm{B}_{\mathrm{Z}}(\mathrm{k}) / \mathrm{q}_{\mathrm{j}}$ and is shown as spectral energy density in Figure $4 \mathrm{~d}$ for proton (blue) and $\mathrm{e}^{-}$(red) masses. In calculating these curves we use the thermal speeds of each species and the observed values of $B_{Z}\left(k_{T}\right)$. Significantly, the spectral energy density in $E_{Z}$ TD, like those found for $E_{Z}$, also peaks at $\mathrm{k}_{\mathrm{T}} \rho_{\mathrm{s}} \approx 1$ where we find the spectral break- point in the magnetic field spectrogram of Figure $4 \mathrm{a}$. We also note that since $E_{Z}$ and $E_{Z}$ TD vary linearly with $k_{Z}$ that $\mathrm{E}_{\mathrm{Z}} / \mathrm{E}_{\mathrm{Z}}$ TD is largely invariant with $\theta_{\mathrm{W}}$. For ions, spectral energy densities an order of magnitude larger in $\mathrm{E}_{Z_{-} \mathrm{TD}}$ than in $E_{Z}$ are found suggesting that TD will be more important than $\mathrm{LD}$ for driving parallel ion energization. For $\mathrm{e}^{-}$we find at $\mathrm{k}_{\mathrm{T}} \rho_{\mathrm{i}} \geq 1$ that $\mathrm{E}_{\mathrm{Z}} \approx \mathrm{E}_{\mathrm{Z}_{-} \mathrm{TD}}$. This result suggests that $\mathrm{e}^{-} \mathrm{LD}$ and TD of KAWs may be of comparable importance in providing the parallel $\mathrm{e}^{-}$acceleration observed.

[12] A consequence of particle acceleration in these waves is the decoupling of the plasma from field-lines and the transport of plasmas from the magnetosheath across the magnetopause into the magnetosphere. The rate at which this occurs may be estimated through a quasi-linear analysis [Hasegawa and Mima, 1978] yielding the diffusion coefficient,

$$
D_{W} \approx\left(\frac{\pi}{8}\right)^{1 / 2} \sum_{k} \frac{k_{y}^{2}}{k_{z}^{2}} \frac{1}{\left|k_{z}\right| v_{i}}\left(\frac{\left|E_{\|}(k)\right|}{B_{o}}\right)^{2} \exp \left(\frac{-V_{A}^{2}}{2 v_{i}^{2}}\right)
$$

where $E_{\|}$is given by $E_{Z}$ from equation (2) for LD. From a consideration of the full ion drift kinetic equation [Johnson and Cheng, 1997] $\mathrm{E}_{\|}$can also be replaced by $\mathrm{E}_{\mathrm{Z}}$ TD where $\mathrm{v}_{\mathrm{i}} \sim \mathrm{V}_{\mathrm{A}}$ for TD. Integrating the results for $\mathrm{D}_{\mathrm{W}}\left(\mathrm{k}_{\mathrm{T}}\right)$ as shown in Figure 4f (LD is black; TD is red) over the observed wavenumber (frequency) range for $\theta_{\mathrm{W}}=89.95^{\circ}$ yields $\mathrm{D}_{\mathrm{W} \text { LD }} \approx 2.6 \times 10^{8}$ and $\mathrm{D}_{\mathrm{W} \text { TD }}=1.2 \times 10^{10} \mathrm{~m}^{2} \mathrm{~s}^{-1}$ while for $88.5^{\circ} \mathrm{D}_{\mathrm{W} L D}=9 \times 10^{6}$ and $\mathrm{D}_{\mathrm{W} \text { TD }}=4.1 \times 10^{8} \mathrm{~m}^{2} \mathrm{~s}^{-1}$. The Bohm rate is $4 \times 10^{8} \mathrm{~m}^{2} \mathrm{~s}^{-1}$. This result shows that cross-field transport in these waves is significant and that TD is more important than LD in driving the diffusion process.

[13] Two other processes may drive cross field transport in these waves. Firstly, drifts on plasma gradients at the magnetopause can couple to the Alfvén waves to provide enhanced transport rates [Johnson and Cheng, 1997]. Quantitative estimates of this contribution require a measurement of these gradients which we have not performed. However, for the observed wave amplitudes and expected gradient scales such effects may be as important as TD. Secondly, the observed amplitudes are sufficient that transport through stochastic ion scattering/demagnetization on $\rho_{\mathrm{i}}$ scale electric field structures may occur. In the single wave case the threshold amplitude required for de-magnetization by this means is given by $\mathrm{E}_{\mathrm{Y}}=\mathrm{B}_{\mathrm{o}} \Omega_{\mathrm{i}} / \mathrm{k}_{\mathrm{y}}$. For $\mathrm{H}^{+}$ions, and the average magnetic field, this threshold is $\sim 5 \mathrm{mV} / \mathrm{m}$ at $\mathrm{k}_{\mathrm{y}} \rho_{\mathrm{i}}=1$ and may be less for the multi-wave case. A consequence of this demagnetization, in addition to facilitating transport, is transverse ion heating [Chen et al., 2001; Johnson and Cheng, 2001]. This in turn increases the magnetic moment and the hence the efficacy of parallel ion acceleration through TD. This combined perpendicular and parallel acceleration process may account for the ion energization observed during the wave burst shown in Figure $2 \mathrm{~g}$ which is primarily transverse at low energies but more isotropic at larger energies where parallel energization through TD becomes more important.

\section{Conclusions}

[14] The low frequency electromagnetic fluctuations observed near the dayside magnetopause from the THEMIS 
spacecraft may be described as a turbulent spectrum of Doppler shifted KAWs. These waves are most intense where $B_{Z \text { GSE }}$ is negative, suggesting an association with reconnection, and have spectral energies densities obeying power-laws where $\mathrm{B}_{\mathrm{x}}^{2}\left(\mathrm{k}_{\mathrm{T}}\right) \propto \mathrm{k}_{\mathrm{T}}^{-1.5}$ and $\mathrm{k}_{\mathrm{T}}^{-3.0}$ for $\mathrm{k}_{\mathrm{T}} \rho_{\mathrm{s}}<1$ and $\mathrm{k}_{\mathrm{T}} \rho_{\mathrm{s}}>1$ respectively. The former power-law is suggestive of a Krachinan-Iroshnikov cascade for isotropic turbulence which may be appropriate for $\mathrm{k}_{\mathrm{T}}<1 / \rho_{\mathrm{s}}$. In the later case, for the range $\mathrm{k}_{\mathrm{T}} \geq 1 / \rho_{\mathrm{s}}$ the observations presented in Figure 4 show that the fluctuations are however anisotropic with $\theta_{\mathrm{W}}$ as large as $89.95^{\circ}$. Over this range the scaling is similar to that reported by Sundkvist et al. [2007] and Sahraoui et al. [2006] in the magnetosheath and by Narita et al. [2006] in the foreshock. We show that over this range these waves may drive particle heating/acceleration/transport through LD, TD and stochastic transverse ion acceleration via demagnetization on gyro-radii scale field structures. TD is likely the most important process for parallel ion energization, while for $\mathrm{e}^{-}$, TD may be comparable to that provided through LD. Calculation of $\mathrm{D}_{\mathrm{W}}$ shows that these waves may be capable of providing transport across the magnetopause at the Bohm rate for $\theta_{\mathrm{W}}>88^{\circ}$ (the $\theta_{\mathrm{W}}$ that best fits observations for $\mathrm{k}_{\mathrm{T}} \geq 1 / \rho_{\mathrm{s}}$ ). However, since $\mathrm{D}_{\mathrm{W}}$ is sensitive to $\theta_{\mathrm{W}}$, the uncertainty in $\theta_{\mathrm{W}}$ means a large uncertainty in $\mathrm{D}_{\mathrm{W}}$.

[15] Acknowledgments. We acknowledge NASA contract NAS502099. Chris Chaston was supported in part by NSF grants ATM0612614, ATM-0602728 and is grateful for comments by Yasuhito Narita. The FGM team receives support through the German Ministry for Economy and Technology and the German Center for Aviation and Space (DLR) under contract 50 OC 030 .

\section{References}

Angelopoulos, V. (2008), The THEMIS mission, Space Sci. Rev., in press. Auster, H. U., et al. (2008), The THEMIS fluxgate magnetometer, Space Sci. Rev., in press.

Chaston, C. C., et al. (2005), Drift-kinetic Alfvén waves observed near a reconnection X-line in the Earth's magnetopause, Phys. Rev. Lett., 95, 065002.

Chaston, C. C., et al. (2007), Mode conversion and anomalous transport in Kelvin-Helmholtz vortices and kinetic Alfvén waves at the Earth's magnetopause, Phys. Rev. Lett., 99, 175004.

Chen, L., Z. Lin, and R. White (2001), On resonant heating below the cyclotron frequency, Phys. Plasmas, 8, 4713.
Hasegawa, A., and K. Mima (1978), Anomalous transport produced by kinetic Alfvén wave turbulence, J. Geophys. Res., 83, 1117.

Johnson, J. R., and C. Z. Cheng (1997), Kinetic Alfvén waves and plasma transport at the magnetopause, Geophys. Res. Lett., 24, 1423.

Johnson, J. R., and C. Z. Cheng (2001), Stochastic ion heating at the magnetopause due to kinetic Alfvén waves, Geophys. Res. Lett., 28, 4421.

Johnson, J. R., et al. (2001), Signatures of mode conversion and kinetic Alfvén waves at the magnetopause, Geophys. Res. Lett., 28, 227.

Lee, L. C., et al. (1994), Kinetic Alfvén waves as a source of plasma transport at the dayside magnetopause, J. Geophys. Res., 99, 17,405.

Lysak, R. L., and W. Lotko (1996), On the kinetic dispersion relation for shear Alfvén waves, J. Geophys. Res., 101, 5085.

McFadden, J. P., C. W. Carlson, D. Larson, V. Angelopolos, M. Ludlam, R. Abiad, and B. Elliot (2008), The THEMIS ESA plasma instrument and in-flight calibration, Space Sci. Rev., in press.

Narita, Y., et al. (2006), Wave-number spectra and intermittency in the terrestrial foreshock region, Phys. Rev. Lett., 97, 191101.

Nishino, M. N., et al. (2007), Geotail observations of temperature anisotropy of the two-component protons in the dusk plasma sheet, Ann. Geophys., 25, 769.

Rezeau, L., et al. (1989), Characterization of Alfvénic fluctuations in the magnetopause boundary layer, J. Geophys. Res., 94, 101.

Sahraoui, F., et al. (2006), Anisotropic turbulent spectra in the terrestrial magnetosheath as seen by the Cluster spacecraft, Phys. Rev. Lett., 96, 075002 .

Song, P., C. T. Russell, T. I. Gombosi, and D. L. DeZeeuw (2003), A model for the formation of the low latitude boundary layer for northward IMF by reconnection: A summary and review, in Earth's Low-Latitude Boundary Layer, Geophys. Monogr. Ser., vol. 133, edited by P. Newell and T. Onsager, p. 121, AGU, Washington, D. C.

Stasiewicz, K., C. E. Seyler, F. S. Mozer, G. Gustafsson, J. Pickett, and B. Popielawska (2001), Magnetic bubbles and kinetic Alfvén waves in the high-latitude magnetopause boundary, J. Geophys. Res., 106, 29,503 .

Sundkvist, D., et al. (2007), Dissipation in turbulent plasma due to reconnection in thin current sheets, Phys. Rev. Lett., 99, 025004.

V. Angelopoulos and C. T. Russell, Institute for Geophysical and Planetary Physics, University of California, Los Angeles, CA 90095, USA.

H. U. Auster and K. H. Glassmeier, Institut für Geophysik und Extraterrestrische Physik, Technische Universität Braunschweig, D-38106 Braunschweig, Germany.

J. Bonnell, C. W. Carlson, C. Chaston, and J. P. McFadden, Space Science Laboratory, University of California, Berkeley, CA 94720-7450, USA. (ccc@ssl.berkeley.edu)

C. Cully, Laboratory for Atmospheric and Space Physics, University of Colorado, Boulder, CO 80303, USA.

O. Le Contel and A. Roux, Centre d'Etude des Environnements Terrestre et Planétaires, F-78140 Velizy, France. 